\title{
SARS-CoV-2 detection in pregnant and non-pregnant women
}

\author{
Noor Hazim Abdulkareem ${ }^{1, *, \dagger}$ (D) , Elham Hazeim Abdulkareem ${ }^{2, \dagger}$ (D) \\ ${ }^{1}$ Department of Obstetrics and Gynecology, University of Anbar, College of Medicine, 55431 Baghdad, Ramadi, Iraq \\ ${ }^{2}$ Department of Oral and Maxillofacial Surgery, University of Anbar, College of Dentistry, 55431 Baghdad, Ramadi, Iraq \\ *Correspondence: den.noor.h@uoanbar.edu.iq (Noor Hazim Abdulkareem) \\ ${ }^{\dagger}$ These authors contributed equally.
}

DOI:10.31083/j.ceog 4805183

This is an open access article under the CC BY 4.0 license (https://creativecommons.org/licenses/by/4.0/).

Submitted: 3 April 2021 Revised: 24 April 2021 Accepted: 19 May 2021 Published: 15 October 2021

Background: The coronavirus causing severe acute respiratory syndrome has infected over 2 million people worldwide, killed thousands, and triggered a global pandemic. There is a scarcity of information about pregnant and non-pregnant women who have coronavirus disease (COVID-19). This study's aim was to determine whether the severe acute respiratory syndrome coronavirus 2 was present in the vaginal fluid of infected women. Also, their platelet counts were examined to see whether they were higher in severe versus moderate cases of the COVID-19 disease. Methods: Our Obstetrics Clinic treated 31 women with confirmed COVID-19 (23 pregnant and 8 non-pregnant women) between 1 September 2020 and 30 September 2020). The existence of syndrome coronavirus 2 (SARS-CoV-2) in vaginal fluid samples was used to determine evidence of genital transmission of the virus. A clinical laboratory procedure, including taking blood samples, IgG/IgM Rapid Test Cassette was performed to validate SARS-CoV-2 infection. Vaginal swabs were retrieved from SARS-CoV-2 positive patients between seven and 10 days of onset. Swabs were inserted two or three centimetres into the vagina and rotated three or five times. The swabs were immediately sent to the lab for RT-PCR, and the patients' COVID-19 status was verified. The real-time Polymerase Chain Reaction (BIORON Diagnostics CmbH's - RealLine SARS-CoV-2 (B-Format) Bl1020-96 Tests). Results: Patients' vaginal fluids tested negative for SARS-CoV-2. Patients who tested positive for SARS-CoV-2 had normal platelet counts. Discussion: Our findings indicate that SARS-2 virus was not present in the vaginal fluid of the 31 patients, meaning that sexual transmission of SARSCoV-2 is unlikely.

\section{Keywords}

COVID-19; Coronavirus infection; Pregnancy; SARS-CoV-2; Thrombocytopenia; Vaginal fluids

\section{Introduction}

In March 2020, the World Health Organization (WHO) announced that a novel coronavirus disease (COVID-19) outbreak was now a pandemic, and it remains a global public health emergency [1]. In symptomatic cases, transmission occurs via a respiratory zoonotic virus. The infection progresses through an asymptomatic incubation period, which is calculated to be approximately two to 10 days [2]. Most patients afflicted with severe acute respiratory syndrome coronavirus 2 (SARS-CoV-2) have an upper respiratory infection.
They can experience a sore throat and fever, although they typically recover without medication. However, symptoms such as pneumonia and tissue hypoxia can become severe. Devastating complications such as organ failure, septic shock, pulmonary oedema, and acute respiratory distress syndrome can result in a life-threatening condition [3].

SARS-CoV-2 has affected about two million people worldwide. It has caused thousands of deaths and has become a worldwide pandemic [3, 4]. SARS is transmitted by airborne particles such as droplets or through direct contact $[5,6]$. It induces inflammation of the lungs that can advance to a cytokine storm in the most extreme cases [6]. It is noteworthy that during pregnancy, cell-mediated immunity is compromised; this results in a decreased resistance to infectious diseases [7].

SARS-CoV-2 has been detected in diverse organs, and in areas such as the throat and the anus; however, it has not been detected in the uterus. Nonetheless, it is uncertain whether SARS-CoV-2 can be transmitted through sexual intercourse. In comparison, Ebola has been found in female patients' vaginal fluids [8]. We focused on pregnant patients diagnosed with COVID-19 since pregnant women may face a higher risk of SARS-CoV-2 infection and may experience more complex health events.

COVID-19 can induce a variety of symptoms and diseases. Certain patients can experience a severe pro-inflammatory syndrome that is consistent with complex coagulopathy and pro-coagulant endothelial phenotypes. Upon initial infection, COVID-19 cells increase fibrinogen and D-dimer levels. This allows fibrinogen degradation products to be elevated. Patients frequently present with moderate thrombocytopenia [9]. Patients diagnosed with COVID-19 can exhibit an abnormally brief prothrombin time and partial thromboplastin time [10]. Elevated D-dimer levels have been linked to higher mortality rates [9]. In viral infections, thrombocytopenia, platelet secretion, and leukocyte interactions either have deleterious or beneficial effects on the body's immune response [11]. A syndrome called thrombocytopenia is found in $6 \%-42 \%$ of confirmed cases of COVID-19. In the 2003 SARS epidemic, $20 \%-55 \%$ of patients infected by the virus ex- 
hibited thrombocytopenia. Thrombocytopenia is also associated with Middle East Respiratory Syndrome (MERS). While certain researchers have considered platelet counts to be a significant indicator of mortality from COVID-19, others have not [12].

Severe pneumonia that causes inflammation of the blood vessels harms the pulmonary arteries and can induce thrombus in the earlier phases of the disease [13]. Despite the administration of standard thromboprophylaxis, the occurrence of venous and perhaps arterial thrombosis remains high in COVID-19 patients [14].

The clinical features and potential for transmission of COVID-19 in pregnant women remains unclear [15]. There is an urgent need to resolve the issues related to COVID-19 in pregnant women. There are concerns whether pregnant women with COVID-19 develop distinct symptoms from those of non-pregnant women. For instance, a non-pregnant woman diagnosed with COVID-19 is more likely to have had a sexually transmission of COVID-19 than is a pregnant woman. Additionally, it could be more likely that a pregnant woman who has been diagnosed with COVID-19 would transmit the virus to her child.

The principles of obstetric treatment for pregnant women with COVID-19 infection must be clarified [16]. In a clinical setting, detailed clinical data from pregnant and nonpregnant women with laboratory-confirmed COVID-19 infection collected and analysed. Despite a rise in COVID-19 cases, there is a scarcity of information on the clinical aspects of COVID-19 in pregnant women. The purpose of this analysis was to investigate whether pregnant women had evidence of SARS-CoV-2 in their vaginal fluid and to analyse their platelet counts.

\section{Materials and methods}

COVID 19 was diagnosed based on the WHO's Global Health Intermediate Recommendations [4]. SARS-CoV-2 was evaluated using the WHO real-time RT-PCR (BIORON Diagnostics GmbH's - RealLine SARS-CoV-2 (B-Format) BI1020-96 Tests) [17-20]. The COVID-19 IgG/IgM Rapid Test Cassette (Biotech, Schiffgraben 41, 30175 Hannover, Germany) was used in this study [21]. A clinical laboratory procedure, including blood samples for COVID-19 rapid tests, was performed. Vaginal swabs were retrieved from SARS-CoV-2 positive patients between seven and 10 days of onset. Verbal permission was requested from all qualifying participants. Qualifying patients were requested to participate in the trial, and all patients who agreed signed consent forms. Ethical approval for the study was obtained from the Research Ethical Committee of the University of Anbar, Iraq, 2020, No. 15. No patients were admitted to the intensive care unit, and none had intrusive ventilation. All patients had the capacity to comply completely with the usual gynaecological examination protocol when the samples were retrieved. They were also able to assume a lithotomy position. Vaginal swabs were inserted $2-3 \mathrm{~cm}$ into the vagina and rotated three to five times. The swabs were immediately submitted to the laboratory for RT-PCR (Technology Quality Innovation, Analytik Jena GmbH, Konrad-Zuse-Strasse, Jena, Germany), and patients were confirmed as having COVID-19. Patients with positive test outcomes were considered laboratory research patients. Two researchers and laboratory staff members reviewed all results.

\section{Results}

A cohort analysis was performed with 23 pregnant and eight non-pregnant women who were treated at our obstetric clinic. All women reported COVID-19 symptoms between 1 September 2020 and 31 September 2020. Many of the patients were Ramadi residents. Patients were aged from 18 to 58 years $($ mean $=38)$. They reported fever $\left(38^{\circ} \mathrm{C}\right)$, followed by fatigue.

Twelve patients' laboratory examinations resulted in an IgG-negative test, and 18 patients had a positive IgM test. One patient had both a negative IgG and IgM tests. Fifteen patients had both a positive IgG and IgM test. Four patients had a negative IgM test.

At the initiation of the illness and the period of vaginal screening, clinical tests were conducted. Thirty-one patients had their vaginal fluids screened for the existence of SARSCoV-2. As shown in Table 1, the platelet count was within the normal range for both pregnant and non-pregnant patients. None of the vaginal fluid samples tested positive for SARS-CoV-2.

\section{Discussion}

Coronavirus causes acute respiratory distress, ranging from asymptomatic or mild symptoms to serious illness resulting in respiratory failure, shock, multi-organ dysfunction, and even death. Data concerning COVID-19 related maternal morbidity are not yet fully developed. They suggest a complex path from asymptomatic disease to minor illness to death [22]. The clinical symptoms of pregnant COVID-19 patients in this study varied considerably from asymptomatic disease to quite serious complications. It was comparable to previous research on non-pregnant patients [23, 24]. The primary symptoms were fever and fatigue, while less frequent symptoms included sore throat and shortness of breath. This was in agreement with a previous study [23].

According to our research, the clinical presence of SARSCoV-2 infection during pregnancy is identical to that of nonpregnant patients, as has been previously reported [4, 24, 25]. Fever was the most common presenting complaint in both pregnancy categories and has been previously defined as the most common presenting complaint overall [4]. The effects of other viral infections on the female reproductive tract have been studied in the past $[26,27]$. For instance, the existence of Zika Virus in women's reproductive tracts was discovered by Prisant et al. [26]. Furthermore, Bausch et al. [28] discovered Ebola virus in the vaginal fluids of a woman with Ebola fever 33 days after the epidemic began. 
Table 1. SARS-CoV-2 in vaginal fluid, IgG, IgM, platelet count, and patient data.

\begin{tabular}{|c|c|c|c|c|c|c|c|}
\hline Patient no. & Age, y & Pregnancy month & $\begin{array}{l}\text { Fatigue, joint pain, } \\
\text { and back pain }\end{array}$ & $\begin{array}{l}\text { SARS-CoV-2 in vaginal fluid } \\
\text { was detected by RT-PCR }\end{array}$ & $\operatorname{IgG}$ & $\operatorname{IgM}$ & Platelet count \\
\hline 1 & 18 & 5 & + & - & - & + & 200 \\
\hline 2 & 36 & - & + & - & - & + & 200 \\
\hline 3 & 20 & 6 & + & - & + & + & 200 \\
\hline 4 & 22 & 3 & + & - & - & + & 200 \\
\hline 5 & 58 & - & Ovarian cyst & - & + & + & 200 \\
\hline 6 & 27 & 8 & + & - & + & + & 176 \\
\hline 7 & 37 & 8 & + & - & - & + & 200 \\
\hline 8 & 29 & 3 & + & - & + & + & 200 \\
\hline 9 & 24 & 5 & + & - & + & + & 243 \\
\hline 10 & 19 & 4 & + & - & + & + & 222 \\
\hline 11 & 42 & 9 & + & - & + & + & 242 \\
\hline 12 & 40 & - & + & - & + & + & 329 \\
\hline 13 & 20 & 9 & + & - & - & + & 187 \\
\hline 14 & 32 & 5 & + & - & + & + & 309 \\
\hline 15 & 30 & 5 & + & - & + & + & 255 \\
\hline 16 & 37 & - & + & - & - & + & 228 \\
\hline 17 & 18 & 6 & + & - & + & - & 259 \\
\hline 18 & 45 & - & + & - & + & + & 200 \\
\hline 19 & 24 & 8 & + & - & + & + & 174 \\
\hline 20 & 22 & 8 & + & - & + & + & 192 \\
\hline 21 & 31 & 4 & + & - & - & + & 195 \\
\hline 22 & 45 & - & + & - & + & - & 200 \\
\hline 23 & 26 & 6 & + & - & + & - & 258 \\
\hline 24 & 25 & 6 & + & - & - & + & 202 \\
\hline 25 & 22 & 8 & Loss of smell and taste & - & - & - & 176 \\
\hline 26 & 20 & 8 & + & - & - & + & 313 \\
\hline 27 & 22 & 6 & + & - & + & + & 197 \\
\hline 28 & 30 & - & + & - & - & + & 200 \\
\hline 29 & 28 & - & Vaginal infection & - & - & + & 747 \\
\hline 30 & 19 & 6 & + & - & + & + & 113 \\
\hline 31 & 21 & 4 & + & - & + & + & 347 \\
\hline
\end{tabular}

IgG, Immunoglobulin G; IgM, Immunoglobulin M; RT-PCR, Real time polymerase chain reaction; Absence of 'Pregnancy month' indicates a non-pregnant patient.

Our study, in accordance with the Hong Kong study, offers an initial view of the continuum of the disease and the outcomes associated with SARS-CoV-2 infection during pregnancy [16]. Based on a smaller sample $(n=10)$ in which no SARS-CoV-2 was found in the vaginal fluid, similar findings have recently been published [16]. The virus was not present in the ovaries or uterus in clinical autopsy reports of SARS patients. Vaginal fluids were not tested in one study [29]. Research on the existence of SARS-CoV-2 in the female reproductive tract is limited. This topic is important as it examines the potential for sexual transmission of the virus. In this study, we included 31 women with COVID-19. Unlike the Ebola and Zika viruses, SARS-CoV-2 was shown to be absent in the vaginal fluids of people who had extreme pneumonia. This study aimed to identify SARS-CoV-2 in the vaginal fluid of pregnant and non-pregnant women. In patients who suffered serious SARS-CoV-2 respiratory symptoms, the swabs from their vaginal fluids showed that the virus was not present. According to this study, the risk of transmitting SARS-CoV-2 to sexual partners through a virus in the vaginal canal is low; however, it may exist at a low level. In one study, 17 women with severe SARS-CoV-2 were examined [30]. However, no infection was found in the amniotic fluid or the blood of the umbilical cord [30].

Preliminary results from China indicated slight thrombocytopenia in COVID-19 patients. This has been shown in more than one-third of non-pregnant mothers [31]. In presymptomatic patients, thrombocytopenia has not yet been confirmed [31]. However, evidence concerning pregnant women with COVID-19 is minimal [31, 32]. Thrombocytopenia may be widespread in pregnant women, affecting as many as $10 \%$ of patients. Late pregnancy is the main cause of gestational thrombocytopenia, which is mild in most cases [31].

Thrombocytopenia in COVID-19 is often a marker of the seriousness of the condition, according to the literature. Platelets were examined in various phases of COVID-19 to see whether they were higher in the severe versus moder- 
ate stages of the disease. In our research, we discovered that platelet counts were unrelated to disease severity, which is consistent with a previous analysis by Le Gouez et al. [31].

The platelet count was as low as 113 cells $/ \mathrm{mL}$ among the thirty-one women in the present study. Therefore, the detection of late pregnancy thrombocytopenia was unlikely. In pregnant women, thrombocytopenia is the most common source of preeclampsia [33]. However, none of our patients met the requirements for this condition. Similar findings have recently been reported in a smaller sample $(n=3)$ in which the number of platelets in one case was low $(40 \times$ $\left.10^{9} / \mathrm{L}\right)$, rendering the diagnosis of thrombocytopenia in late pregnancy impossible [34]. This is what our analysis observed in serious as well as mild cases of COVID-19. Since many pregnant women with low clinical risk have elevated markers and many pregnant women with very high clinical risk have regular markers, we did not include prothrombin period, partial thromboplastin time, or D-dimer in our research [35].

It is necessary to consider the potential limitations to this study. The number of patients was limited. However, a significant point to consider is the strength of our research. It is difficult to conduct research with pregnant patients, especially during a pandemic. Accordingly, data are lacking regarding COVID-19 and pregnancy. Women who participated in this research represented all three trimesters of pregnancy. This study examined typical SARS-CoV-2 symptoms. Laboratory tests for pregnant and non-pregnant patients with temperature, fatigue, joint pain, and cough were conducted.

\section{Conclusions}

In conclusion, our research found that pregnant women are indeed vulnerable to infection with SARS CoV-2. Efforts should be made to minimize the rate of infection with SARSCoV-2 during pregnancy and the perinatal periods. Additional comprehensive care is required for pregnant women. This analysis found no evidence of SARS-CoV-2 during testing for SARS-CoV-2 in the vaginal fluids of pregnant and non-pregnant women.

\section{Abbreviations}

COVID-19, novel coronavirus disease; SARS-CoV-2, severe acute respiratory syndrome coronavirus 2 .

\section{Author contributions}

Conception and design of study-NHA. Acquisition of data-NHA. Analysis and/or interpretation of data-NHA. Drafting the manuscript-EHA. Revising the manuscript critically for important intellectual content-NHA and EHA. Approval of the version of the manuscript to be publishedNHA and EHA. All authors contributed to editorial changes in the manuscript. All authors read and approved the final manuscript.

\section{Ethics approval and consent to participate}

Ethical approval for the study was obtained from the Research Ethical Committee of the University of Anbar, Iraq 2020, Ref. 15 on 16-11-2020. Informed consent was obtained from all participants at the time of enrollment.

\section{Acknowledgment}

We would like to extend our gratitude to the University of Anbar, the Ministry of Health and the Ministry of Higher Education and Scientific Research in Iraq for their support.

\section{Funding}

This research received no external funding.

\section{Conflict of interest}

The authors declare no conflict of interest.

\section{References}

[1] Hulswit RJ, de Haan CA, Bosch BJ. Coronavirus spike protein and tropism changes. Coronaviruses. 2016; 20: 29-57.

[2] Rothe C, Schunk M, Sothmann P, Bretzel G, Froeschl G, Wallrauch $\mathrm{C}$, et al. Transmission of 2019-nCoV infection from an asymptomatic contact in Germany. New England Journal of Medicine. 2020; 382: 970-971.

[3] Phan T. Novel coronavirus: from discovery to clinical diagnostics. Infection, Genetics and Evolution. 2020; 79: 104211.

[4] Huang C, Wang Y, Li X, Ren L, Zhao J, Hu Y, et al. Clinical features of patients infected with 2019 novel coronavirus in Wuhan, China. Lancet. 2020; 395: 497-506.

[5] Wool G, Miller J. The Impact of COVID-19 disease on platelets and coagulation. Pathobiology. 2020; 88: 15-27.

[6] Varghese G, John R, Manesh A, Karthik R, Abraham O. Clinical management of COVID-19. Indian Journal of Medical Research. 2020; 151: 401.

[7] Jamieson D, Theiler R, Rasmussen S. Emerging infections and pregnancy. Emerging Infectious Diseases. 2006; 12: 1638-1643.

[8] Green E, Hunt L, Ross JCG, Nissen NM, Curran T, Badhan A, et al. Viraemia and Ebola virus secretion in survivors of Ebola virus disease in Sierra Leone: a cross-sectional cohort study. The Lancet Infectious Diseases. 2016; 16: 1052-1056.

[9] Connors JM, Levy JH. COVID-19 and its implications for thrombosis and anticoagulation. Blood. 2020; 135: 2033-2040.

[10] Goldenberg NA, Sochet A, Albisetti M, Biss T, Bonduel M, Jaffray $\mathrm{J}$, et al. Consensus-based clinical recommendations and research priorities for anticoagulant thromboprophylaxis in children hospitalized for COVID-19-related illness. Journal of Thrombosis and Haemostasis. 2020; 18: 3099-3105.

[11] Assinger A. Platelets and infection-an emerging role of platelets in viral infection. Frontiers in Immunology. 2014; 5: 649.

[12] Hottz ED, Bozza FA, Bozza PT. Platelets in immune response to virus and immunopathology of viral infections. Frontiers in Medicine. 2018; 5: 121.

[13] Al-Samkari H, Karp Leaf RS, Dzik WH, Carlson JCT, Fogerty AE, Waheed A, et al. COVID-19 and coagulation: bleeding and thrombotic manifestations of SARS-CoV-2 infection. Blood. 2020; 136: 489-500.

[14] Al-Ani F, Chehade S, Lazo-Langner A. Thrombosis risk associated with COVID-19 infection. a scoping review. Thrombosis Research. 2020; 192: 152-160.

[15] Zhu N, Zhang D, Wang W, Li X, Yang B, Song J, et al. A novel coronavirus from patients with pneumonia in China, 2019. The New England Journal of Medicine. 2020; 382: 727-733. 
[16] Qiu L, Liu X, Xiao M, Xie J, Cao W, Liu Z, et al. SARS-CoV-2 is not detectable in the vaginal fluid of women with severe COVID19 Infection. Clinical Infectious Diseases. 2020; 71: 813-817.

[17] He F, Deng Y, Li W. Coronavirus disease 2019: what we know? Journal of Medical Virology. 2020; 92: 719-725.

[18] World Health Organization. WHO coronavirus disease (COVID-19) technical guidance: laboratory testing for 2019-nCoV in humans. 2020. Available at: https://www.who.int/emergencies/diseases/novel-coronav irus-2019/technical-guidance/laboratory-guidance (Accessed: 2 March 2020).

[19] World Health Organization. Molecular assays to diagnose COVID-19. Coronavirus disease (COVID-19) technical guidance: laboratory testing for $2019-\mathrm{nCoV}$ in humans. 2020. Available at: https://www.who.int/publications/i/item/diagnostic-testing -for-sars-cov-2 (Accessed: 11 September 2020).

[20] World Health Organization. Laboratory testing for coronavirus disease 2019 (COVID-19) in suspected human cases. 2020. Available at: https://www.who.int/publications-detail/laborato ry-testing-for-2019-novel-coronavirus-in-suspected-human-c ases-20200117 (Accessed: 4 February 2020).

[21] HEALGEN. COVID-19 IgG/IgM rapid test cassette ( $\mathrm{w}$ hole blood/serum/plasma): instructions for use. 2020. Available at: https://www.fda.gov/media/138438/download (Accessed: 1 July 2020).

[22] Bhatraju PK, Ghassemieh BJ, Nichols M, Kim R, Jerome KR, Nalla AK, et al. Covid-19 in critically ill patients in the seattle regioncase series. New England Journal of Medicine. 2020; 382: 2012 2022.

[23] Xu XW, Wu XX, Jiang XG, Xu KJ, Ying LJ, Ma CL, et al. Clinical findings in a group of patients infected with the 2019 novel coronavirus (SARS-Cov-2) outside of Wuhan, China: retrospective case series. British Medical Journal. 2020; 368: m606.

[24] Liu Y, Chen H, Tang K, Guo Y. Withdrawn: clinical manifestations and outcome of SARS-CoV-2 infection during pregnancy. The Journal of infection. 2020; S0163-4453(20)30109-2.

[25] Chen L, Li Q, Zheng D, Jiang H, Wei Y, Zou L, et al. Clinical characteristics of pregnant women with Covid-19 in Wuhan, China The New England Journal of Medicine. 2020; 382: e100.
[26] Prisant N, Bujan L, Benichou H, Hayot PH, Pavili L, Lurel S, Herrmann C, et al. Zika virus in the female genital tract. The Lancet Infectious Diseases. 2016; 16: 1000-1001.

[27] Visseaux B, Mortier E, Houhou-Fidouh N, Brichler S, Collin G, Larrouy $\mathrm{L}$, et al. Zika virus in the female genital tract. The Lancet Infectious Diseases. 2016; 16: 1220.

[28] Bausch D, Towner J, Dowell S, Kaducu F, Lukwiya M, Sanchez A, et al. Assessment of the risk of Ebola virus transmission from bodily fluids and fomites. The Journal of Infectious Diseases. 2007; 196: S142-S147.

[29] Ding Y, He L, Zhang Q, Huang Z, Che X, Hou J, et al. Organ distribution of severe acute respiratory syndrome (SARS) associated coronavirus (SARS-CoV) in SARS patients: implications for pathogenesis and virus transmission pathways. The Journal of Pathology. 2004; 203: 622-630.

[30] Chen H, Guo J, Wang C, Luo F, Yu X, Zhang W, et al. Clinical characteristics and intrauterine vertical transmission potential of COVID-19 infection in nine pregnant women: a retrospective review of medical records. Lancet. 2020; 395: 809-815.

[31] Le Gouez A, Vivanti AJ, Benhamou D, Desconclois C, Mercier FJ. Thrombocytopenia in pregnant patients with mild COVID-19. International Journal of Obstetric Anesthesia. 2020; 44: 13-15.

[32] Lippi G, Plebani M, Henry BM. Thrombocytopenia is associated with severe coronavirus disease 2019 (COVID-19) infections: meta-analysis. Clinica Chimica Acta. 2020; 506: 145-148.

[33] Cines DB, Levine LD. Thrombocytopenia in pregnancy. Blood. 2017; 130: 2271-2277.

[34] Royal College of Obstetricians and Gynaecologists. Coronavirus (COVID-19) infection in pregnancy information for healthcare professionals. 2020. Available at: https://www.rcog.org.uk/en/g uidelines-research-services/guidelines/coronavirus-pregnancy/ (Accessed: 25 April 2020).

[35] Bombeli T, Raddatz-Mueller P, Fehr J. Coagulation activation markers do not correlate with the clinical risk of thrombosis in pregnant women. American Journal of Obstetrics and Gynecology. $2001 ; 184$ : 382-389. 